\title{
Weiterbildungskonzept Rheumatologie
}

\author{
Das Berner Modell
}

Ch. Beyeler

Das unter einem Leitbild stehende Weiterbildungskonzept unterstützt die zielgerichtete Vorbereitung, Durchführung und Auswertung der Weiterbildung zum angestrebten Facharzt. Die Selbsteinschätzung, allenfalls ergänzt durch eine Fremdeinschätzung, des Wissens, der Fertigkeiten und Einstellungen sowie die Formulierung der allgemeinen und spezifischen beruflichen Ziele erlauben die Ableitung der Weiterbildungsziele, welche an unserer Klinik oder an einer anderen Weiterbildungsstätte erreicht werden können. Der Lernende wird unterstützt durch einen Tutor, einen Mentor, die direkten Weiterbildner und die Weiterbildungsverantwortliche der Klinik. Die Weiterbildungsinhalte des obligatorischen Kerncurriculums und des individuell wählbaren Mantelcurriculums werden strukturiert an organisierten Veranstaltungen und in individuellen Lernsituationen erworben und fortlaufend dokumentiert. Zudem wird der Lernende ermuntert, seinen Lernprozess zu überdenken und unter Zuhilfenahme eines Portfolio festzuhalten.

\section{Das Leitbild}

Das vorliegende Weiterbildungskonzept soll den Facharztanwärtern und den Weiterbildnern als Orientierungshilfe dienen bei der Planung, Implementierung und Evaluation der Weiterbildung vor der Bewerbung um eine Weiterbildungsstelle, bei Stellenantritt und während der Tätigkeit an der Weiterbildungsstätte. Das Berner Modell orientiert sich an Grundsätzen der Erwachsenenbildung und steht unter folgendem Leitbild:

Der Facharztanwärter bereitet sich während seiner Weiterbildung in Rheumatologie zielgerichtet auf seine angestrebte spätere Tätigkeit in Praxis, Spital, Universität oder anderer Institution vor. Dazu erwirbt er sich Fachwissen; Kompetenzen der Problemlösung und Entscheidungsfindung; Kommunikations- und interventionelle Fertigkeiten; Einstellungen gegenüber Patienten, Angehörigen und allen an der Patientenbetreuung beteiligten Berufsgruppen; Respektierung der eigenen Grenzen. Er erarbeitet sich die Lerninhalte auf strukturierte Weise an organisierten Veranstaltungen und in individuellen Lernsituationen unter Benutzung aller ihm zur Verfügung stehenden Lernquellen. Er wird in \footnotetext{
seinem Lernprozess durch die Weiterbildner seinem persönlichen Lernstil entsprechend gefördert durch Lernzielformulierung, Vereinbarung spezifischer Massnahmen, kontinuierliche formative Evaluation und Vorbereitung auf die summative Evaluation.

Korrespondenz:
PD Dr. med. Christine Beyeler, MME Klinik für Rheumatologie und Klinische Immunologie/Allergologie Universitätsspital

E-Mail: christine.beyeler@insel.ch

summative Evaluation.
}

\section{Formulieren von individuellen Weiterbildungszielen}

Das Weiterbildungskonzept basiert auf dem Weiterbildungsprogramm Rheumatologie FMH, welches das Fach Rheumatologie umschreibt, die Ziele, Dauer, Gliederung und Lerninhalte der Weiterbildung festlegt, das Prüfungsreglement beinhaltet sowie die Kriterien für die Einteilung der Weiterbildungsstätten regelt. Die Zielgruppe umfasst Anwärter auf die Facharzttitel Rheumatologie, Physikalische Medizin und Rehabilitation, Innere Medizin sowie Allgemeinmedizin. Die Anstellungsdauer an unserer Klinik ist abhängig von den Vorkenntnissen, dem angestrebten Facharzttitel, den Qualifikationen während der Weiterbildung und den Bedürfnissen der Klinik.

Vor Stellenantritt erstellt der Lernende eine Selbsteinschätzung seiner Kenntnisse und Fertigkeiten anhand einer Checkliste, welche auf dem Themenkatalog der Weiterbildung Rheumatologie basiert und reflektiert seine Einstellungen (Ist-Zustand). Er formuliert seine allgemeinen Ziele hinsichtlich seiner angestrebten späteren beruflichen Funktion und seiner spezifischen beruflichen Aufgaben, beziehungsweise seiner Orientierungsphase bezüglich Facharzttitel oder späterer Tätigkeit (Soll-Zustand). Daraus leitet er gemeinsam mit der Weiterbildungsverantwortlichen der Klinik die individuellen Weiterbildungsziele bezüglich seiner beruflichen Tätigkeiten ab. Diese Weiterbildungsziele lassen sich entweder während der Anstellung an unserer Klinik realisieren oder können während einer späteren Tätigkeit an einer anderen Weiterbildungsstätte erreicht werden. Der Lernende muss sich bewusst sein, dass die Epidemiologie der Erkrankungen, wie er sie an unserem universitären Tertiärzentrum antrifft, nicht unbedingt vergleichbar ist mit der Häufigkeit und dem Schweregrad der Erkrankungen, die er in seiner angestrebten späteren Tätigkeit abklären und behandeln wird. Es ist empfehlenswert, diesen Aspekt bei der Stellenplanung zu berücksichtigen und bei Bedarf bewusst eine andere Weiterbildungsstätte als Ergänzung auszuwählen. Durch die Formulierung der Weiterbildungsziele ist der Lernende in der Lage, seinen individuellen Lernplan zu 
erstellen mit Auswahl der Lerninhalte und Lernsituationen, welche seinem individuellen Lernstil entsprechen.

Der Weiterbildungsvertrag in Ergänzung zum Anstellungsvertrag stellt eine persönliche, in gegenseitigem Einvernehmen ausgehandelte Vereinbarung zwischen dem Lernenden und der Weiterbildungsstätte, vertreten durch dessen Leiter und deren Weiterbildungsverantwortliche, dar. Er beinhaltet Angaben über die bisherige Weiterbildung, aktuelle Kenntnisse, Fertigkeiten und Einstellungen, allgemeine Ziele, spezifische Weiterbildungsziele, spezifische Massnahmen und Kenntnisnahme des Vorgehens bezüglich der formativen und summativen Evaluationen an unserer Klinik inklusive der verwendeten Evaluationsformulare. Besondere Beachtung verdient die Offenlegung des Zielkonfliktes zwischen den Weiterbildungsbedürfnissen des Lernenden und dem Bedarf an ärztlicher Arbeitskraft an unserer Klinik.

\section{Den Lernenden begleiten und unterstützen}

Während seiner praktischen Tätigkeit als Facharztanwärter wird er unterstützt durch einen Tutor, welcher ihn in die praktischen Belange des Arbeitsortes einführt, damit er sich rasch in den Arbeitsabläufen zurechtfindet und die ihm übertragenen Aufgaben optimal erfüllt. Eine Checkliste dient dazu, die Vollständigkeit der Einführung zu überprüfen. Ein Mentor hilft ihm bei der reibungslosen Integration in die Klinik, macht ihn mit den geschriebenen und ungeschriebenen Regeln des Betriebes vertraut und steht ihm als neutraler Ansprechpartner zur Verfügung.

Die direkten Weiterbildner umfassen die Kaderärzte, welche die ärztlichen Tätigkeiten supervidieren und die Qualitätssicherung gewährleisten, das nichtärztliche Team, bestehend aus Pflegenden, Physiotherapeuten, Ergotherapeuten und Sozialarbeitern, welche im klinischen Alltag wie auch während organisierter Weiterbildungsveranstaltungen ihr Wissen und Können den Lernenden weitergeben, sowie die anderen Facharztanwärter unserer Klinik, welche gegenseitig Wissen, Fertigkeiten und Einstellungen austauschen im Sinne eines peer teaching. Alle an der Weiterbildung Beteiligten ermöglichen durch regelmässiges Feedback die Weiterentwicklung (Fachwissen; Kompetenzen der Problemlösung und Entscheidungsfindung; Kommunikations- und interventionelle Fertigkeiten; Einstellungen gegenüber Patienten, Angehöri- gen und allen an der Patientenbetreuung beteiligten Berufsgruppen; Respektierung der eigenen Grenzen).

Zudem steht die Weiterbildungsverantwortliche als Coach zur Verfügung, um die Lernfortschritte zu erörtern, weitere Lernziele zu formulieren und zusätzlichen Unterstützungsbedarf auszuloten. Sie unterstützt den Lernprozess, wonach konkrete Erfahrungen des Lernenden das reflektierende Beobachten stimulieren, die abstrakte begriffliche Erfassung der Erfahrungen bewirken, das aktive Experimentieren auslösen und das Sammeln weiterer konkreter Erfahrungen fördern. Innerhalb des ersten Anstellungsmonates beobachtet sie die Anamneseerhebung und die klinische Untersuchung (Fremdeinschätzung) einerseits zur Klärung des Lernbedarfs, andererseits zur Bestätigung der grundlegenden Kompetenzen. Sie ist besorgt dafür, dass die direkten Weiterbildner über die Bedürfnisse und Weiterbildungsziele des Lernenden informiert sind, damit die Arbeitssituationen optimal als Lernsituationen genutzt werden können. Anhand eines Weiterbildungsthemenkataloges stellt sie sicher, dass die Weiterbildungsinhalte des obligatorischen Kerncurriculums systematisch behandelt werden und die Möglichkeiten des individuell wählbaren Mantelcurriculums allen Beteiligten bekannt sind. Zudem sorgt sie dafür, dass die organisierten Lernveranstaltungen, individuellen Lernsituationen und Lernquellen das ausgewogene Erreichen der kognitiven, affektiven und psychomotorischen Lernziele ermöglichen und den verschiedenartigen Lernstilen gerecht werden. Schlussendlich stellt sie in enger Zusammenarbeit mit dem Planungsverantwortlichen der Klinik sicher, dass der Zielkonflikt zwischen den Weiterbildungsbedürfnissen des Lernenden und dem Bedarf an ärztlicher Arbeitskraft der Klinik konstruktiv angegangen und ausreichend Weiterbildungszeit gewährt wird unter der Voraussetzung einer durchschnittlichen Leistungsfähigkeit des Assistenzarztes. Insbesondere gilt es festzulegen, ob der Lernende regelmässiger Freistellung zur Durchführung von Interventionen unter Aufsicht bedarf (beispielsweise Ultraschall, epidurale Infiltrationen, Intervertebralgelenksinjektionen, Muskelbiopsien, Synoviabiopsien) oder blockweise Absenzen für den Besuch von Kursen und Kongressen beansprucht (beispielsweise Manualmedizin, Evaluation der funktionellen Leistungsfähigkeit, Strahlenschutz, Führungsausbildung, didaktische Ausbildung). Eine administrative Richtlinie regelt die Freistellung und Finanzierung interner und externer Weiterbildungsveranstaltungen während der Arbeitszeit. 


\section{Reflektieren und evaluieren}

Die Lernsituationen umfassen organisierte Veranstaltungen wie Vorträge, Seminare, Kurse, Kurzdemonstrationen, Fallbesprechungen und Röntgenrapporte, welche vorwiegend dem Lehrerzentrierten, aktivierenden systematischen Lernen dienen. Die individuellen Lernsituationen umfassen die Supervision durch die direkten Weiterbildner während Patientenvorstellungen, Interventionen und Visiten sowie das Selbststudium, wo das Lernende-zentrierte und Gelegenheitslernen im Vordergrund stehen. Die besuchten Lernveranstaltungen und die durchgeführten Eingriffe werden fortlaufend notiert. Zudem wird der Lernende ermuntert, seinen Lernprozess zu überdenken und unter Zuhilfenahme eines Portfolio zu dokumentieren. Dieses ist eine Sammlung von Materialien mit Schlüsselereignissen, welche das reflektierende Lernen und nicht ausschliesslich das Sammeln von Erfahrungen illustrieren. Der Lernende kann darüber entscheiden, was er in sein Portfolio einschliessen will und wer wann Einblick in welche Dokumente erhält.

Die Evaluationen der Lernenden erfolgen formativ durch regelmässiges Feedback durch alle an der Weiterbildung Beteiligten und formelle Gespräche mit der Weiterbildungsverantwortlichen sowie summativ durch Mitarbeitergespräche des Bereichsleiters gemeinsam mit einem direkten Weiterbildner und die Facharztprüfung. Dabei achten wir auf eine klare personelle Trennung zwischen der formativen und summativen Evaluation und beziehen die Weiterbildungsverantwortliche bewusst nicht in Gespräche von selektionierendem Charakter ein. Die Evaluation der Weiterbildungsstätte erfolgt zur
Zeit formativ mittels formeller und informeller Gespräche. Es sind alle eingeladen, Veränderungsvorschläge vorzubringen, mit anderen Lernenden oder Weiterbildnern zu erörtern sowie die Umsetzung der Verbesserungsmassnahmen mitzutragen. In Zukunft sind Visitationen durch ein Visitationsteam, bestehend aus einem Delegierten der Fachgesellschaft, einem Vertreter der Vereinigung Schweizerischer Assistenz- und Oberärzte VSAO sowie einem von der Kommission Weiter- und Fortbildung KWFB bestimmten unabhängigen Experten im Sinne summativer Evaluationen mit Akkreditierung der Weiterbildungsstätte, vorgesehen.

\section{Ausblick}

Das Weiterbildungskonzept wird regelmässig revidiert zwecks Anpassungen an die gesammelten Erfahrungen, an veränderte gesundheitspolitische Bedingungen oder übergeordnete Bestimmungen der Fachgesellschaften beziehungsweise der Vereinigung der Schweizer Ärztinnen und Ärzte (FMH). Dieser kontinuierliche Prozess mit dem Ziel der Qualitätssteigerung der Weiterbildung in Rheumatologie hat jederzeit allgemeine Bedürfnisse, die Bedürfnisse der Weiterzubildenden, die Lernziele, die Lern- und Lehrstrategien, die Implementierung und Evaluation im Auge zu behalten. Die Umsetzung muss Schritt für Schritt und koordiniert erfolgen unter Berücksichtigung der Aspekte von Dienstleistung, Ausbildung, Fortbildung, klinischer Forschung, Organisationsstrukturen und Kulturen aller Beteiligter. Die Qualität der Weiterbildung von heute wird über die Qualität der ärztlichen Versorgung in der Zukunft entscheiden. 